\title{
Gamificación en la universidad: una experiencia basada en el "bring your own device” en educación superior
}

\section{Teresa San-Miguel, Javier Megías y Eva Serna}

Departament de Patologia, Facultat de Medicina i Odontologia, Universitat de València. Teresa SanMiguel (teresa.miguel@uv.es), Javier Megías (javier.megías@uv.es), Eva Serna (eva.serna@uv.es)

\begin{abstract}
Technological development offers several new opportunities to improve the quality of teaching. Under the ongoing task as a teachers of motivating and teaching how to learn to our students, we present the results of a session that consisted of two gamificated and groupal activities (quiz format) and a single self-assessment, based all of them on the use of their own electronic enabled devices (bring your own device). This implies an enriching, fun and practical experience to approach the course, and it provided interesting feedback to continue implementing new technologies at the university.
\end{abstract}

Keywords: gamification, motivation, efficiency, bring your own device, quiz

\begin{abstract}
Resumen
El desarrollo tecnológico ofrece infinidad de nuevas posibilidades para mejorar la calidad de la docencia. Bajo la continua tarea como docentes de motivar y enseñar a aprender a los alumnos, presentamos los resultados de una sesión que constó de dos actividades grupales gamificadas (formato concurso) y una individual de autoevaluación, fundamentadas en el uso de sus propios dipositivos electrónicos (bring your own device). Supuso una experiencia enriquecedora, divertida y práctica para el abordaje de la asignatura, y nos proporcionó un interesante "feedback" para continuar implementando las nuevas tecnologías en la universidad.
\end{abstract}

Palabras clave: gamificación, motivación, rendimiento, “bring your own device”, concurso

\section{Introducción}

A pesar de la estructuración de los planes de estudios en el sistema de grado/postgrado y de la implantación de los créditos ECTS, la universidad continúa trabajando en su adaptación real al Espacio Europeo de Educación Superior (EEES) y lo que implica. Este sistema supone, por una parte, mayor peso del trabajo autónomo de los estudiantes, pero es fundamental el papel del docente, al que se le insta a diversificar las actividades empleadas para el aprendizaje, recuperar la tutoría como herramienta, no sólo didáctica, sino también pedagógica, y de una manera cada vez más necesaria, a utilizar las nuevas tecnologías en su labor docente (Tejedor 2007). Sobre esto último, debemos destacar que "aplicar nuevas tecnologías" no significa simplemente informatizar el aula y utilizar presentaciones 
proyectadas frente a la tradicional pizarra, sino introducir, de manera continuada, novedades tecnológicas que capten la atención de un alumnado que crece en una sociedad hiperestimulada, de cara a favorecer su implicación con las asignaturas y su motivación para conocerlas y comprenderlas, adquiriendo las competencias necesarias para ello.

En este sentido, aunque los componentes que influyen sobre la motivación son numerosos y muy diversos, debemos estimular al alumno para que se haga cargo de sí mismo y de aprender a aprender, aspectos que son críticos en el EEES (Martínez-González 2011); para estas tareas, la motivación es un elemento clave. Además, el bajo rendimiento universitario descrito en los países de nuestro entorno económico-cultural, valorado a través de las tasas de abandono-éxito, de regularidad académica o de las calificaciones obtenidas (Tejedor 2007) es un problema al que hacer frente y para el que debemos recurrir a nuevas estrategias didácticas. Este rendimiento, en términos generales viene definido por cinco tipos de variables según el citado trabajo (Tejedor 2007):

- Variables de identificación (género, edad)

- Variables psicológicas (aptitudes intelectuales, personalidad, motivación, estrategias de aprendizaje, etc.)

- Variables académicas (tipos de estudios cursados, curso, opción en que se estudia una carrera, rendimiento previo, etc.)

- Variables pedagógicas (definición de competencias de aprendizaje, metodología de enseñanza, estrategias de evaluación, etc.)

- Variables socio-familiares (estudios de los padres, profesión, nivel de ingresos, etc.)

Si bien en nuestra mano como docentes, está el actuar sobre las variables pedagógicas, modificando nuestras metodologías de enseñanza, las acciones que tomemos podrán impactar de manera directa sobre la motivación y el aprendizaje de los alumnos. Integrando la utilización de las nuevas tecnologías e interrumpiendo la estructura clásica del aula, podemos lograr nuevas respuestas por parte del estudiante, ayudándole en la contextualización de los contenidos y repercutiendo directamente sobre su implicación. Contreras y sus colaboradores aseguran que el profesor, que es un entrenador, puede incidir favorablemente en la motivación intrínseca, la auto-confianza y el estado de ánimo de sus alumnos (Contreras 2004).

Así, surgió la utilización de los Personal response system (PRS) adaptados a la docencia; estos consistían en la utilización de clickers gracias a los cuales el docente elabora una pregunta y los asistentes contestan a tiempo real. No obstante este sistema plantea algunos problemas: requiere de un software específico que ha de ser instalado en cada aula en la cual vaya a realizarse el concurso, además hay que solicitar los pulsadores y el sistema infrarrojo de detección al centro correspondiente de la universidad y el docente ha de familiarizarse con el programa en cuestión. Aunque el sistema recuerda al mítico programa “¿Quiere ser millonario?”, favoreciendo la participación del alumnado, los requisitos técnicos dificultan su implementación en nuestro sistema educativo.

(cc) EY-NC-ND 2016, Universitat Politècnica de València 
Según el informe NMC Horizon Report 2016 Higher Education Edition, es inexorable la utilización de estrategias basadas en el Bring Your Own Device (BYOD); fundamentado en que cada vez más personas llevan sus propios ordenadores portátiles, tabletas, teléfonos inteligentes y otros dispositivos portátiles, se plantea que estos dispositivos puedan utilizarse para aprender, sustituyendo a los $P R S$ clásicos.

Bajo esta premisa, surgen diversas alternativas recientes al método de clickers, de las que nos centraremos en 2: por una parte, en los últimos años se ha acercado a las aulas universitarias el software SOCRATIVE (www.socrative.com). Se trata de un programa que permite elaborar preguntas y recibir las respuestas de manera inmediata, corrigiendo al participante si lo requiere. Su principal ventaja es la utilización de cualquier dispositivo portátil del usuario para responder e igualmente recibir feedback inmediato en su dispositivo antes de proseguir con la actividad. Además incluye explicaciones en texto que son introducidas por el docente cuando elabora el cuestionario. Por otra parte, para simplificar igualmente los recursos necesarios para hacer un test en el aula, pero además gamificarlo, haciéndolo más atractivo para los estudiantes, el profesor de la Norwegian University of Science and Technologyse, Alf Inge Wang, desarrolló en 2013 un software on-line que permite hacer quizes (concursos), encuestas y debates, con el nombre de KAHOOT (https://kahoot.it/). El elemento ludificante que incluye el programa, es la clasificación pública que establece tras la resolución de cada pregunta y no sólo el feedback con la respuesta correcta (Holguín 2014). Esta ordenación de los participantes, considera la rapidez y la veracidad de las respuestas. Su base pedagógica trata de clasificar a los participantes animándolos a superarse a sí mismos y a sus compañeros: aparecen en pantalla únicamente los 6 mejores participantes, de manera que los primeros clasificados compiten para lograr el primer puesto, mientras los demás participantes se esfuerzan por mejorar en sus respuestas para aparecer en la clasificación. En cierto modo, es la manera de introducir el factor "puntos" de cualquier videojuego clásico, a la actividad del aula (Cortizo 2011). El programa es de acceso libre y puede utilizarse desde la red con cualquier dispositivo con conexión a internet.

Este método de gamificación en el aula es todavía infrecuente en nuestra universidad, pero representa una herramienta potencial para impulsar cambios de hábito tanto en los estudiantes, con un papel más activo en el proceso de enseñanza-aprendizaje, como en los profesores, modificando su paradigma de la docencia, que son entre otras, las metas que persigue el EEES (Mauricio 2015). La gamificación motiva al alumno incrementando su dedicación a preparar y resolver la propuesta que diseña el docente y mejora su predisposición a adquirir las competencias derivadas de la asignatura (Kapp 2012, Zichermann 2011). Aplicada a la docencia universitaria, la introducción del juego en el aprendizaje supone una oportunidad para incentivar la participación del alumnado desde la motivación, tal y como plantean Piñeiro-Otero y sus colaboradores (Piñeiro-Otero 2015), al crear un espacio de conocimiento compartido que resulta muy provechoso para el estudiante y que en último término, favorece la mejora de la calidad de la enseñanza que pretende promover el EEES.

(cc) EY-NC-ND 2016, Universitat Politècnica de València

Congreso In-Red (2016) 
En base a todo lo descrito, nos planteamos desarrollar una experiencia que nos permitiera familiarizarnos con estos sistemas $P S R$, que mejoran la participación en el aula frente a la vía de respuesta oral, basados en $B Y O D$ lo que limita la distracción que supone la tecnología que llevan los propios estudiantes. Además nos proponemos comparar una versión gamificada para incrementar la motivación para "estudiar, aprender a aprender y aprender a resolver", frente a un software más clásico.

\section{Objetivos}

El principal objetivo de este trabajo es describir la valoración que hace un grupo de alumnos universitarios del Grado de Ingeniería Biomédica (UPV-UV) sobre su experiencia de gamificación en el aula, el impacto que podría tener sobre sus resultados y sus preferencias.

La asignatura Morfología a nivel celular y tisular del Grado de Ingeniería Biomédica se estructura en dos unidades didácticas: una basada en la Biología Celular y otra basada en la Estructura Tisular. Las unidades didácticas se evalúan de forma separada de manera que, tras 6 semanas de Morfología a nivel celular, finaliza la docencia de esa parte y comienza la docencia de la siguiente. Un mes después, se evalúa en primera convocatoria Morfología a nivel celular, mediante un examen tipo test de 40 preguntas.

La tasa de alumnos que aprueban en primera convocatoria ha sido del $54 \%$ en los cursos 2013-2014 y 2014-2015. Tasa que asciende hasta el $83 \%$ en la segunda convocatoria y los principales problemas que refieren los alumnos durante la revisión de exámenes son:

- Dificultad para la comprensión de enunciados por la presión del examen.

- Falta de previsión de tiempo para preparar la asignatura.

- Carencias conceptuales previas.

Con la finalidad de introducir nuevas experiencias en el aula para paliar estas dificultades, desarrollamos una sesión práctica que incluía 6 subobjetivos concretos:

1. Motivar a los alumnos para preparar parte del temario con antelación a la primera convocatoria de la asignatura.

2. Garantizar el conocimiento de conceptos básicos mediante un concurso en grupos pequeños de nivel "iniciación".

3. Afianzar los conceptos explicados en clase mediante un concurso en grupos pequeños al nivel de los contenidos.

4. Permitir a los alumnos autoevaluarse mediante cuestionario individual interactivo.

5. Mostrar a los estudiantes preguntas tipo test elaboradas por el profesorado que imparte la asignatura, reduciendo así su nivel de estrés ante el examen.

6. Conocer la impresión de los alumnos sobre la utilización de actividades interactivas de tipo gamificación.

(cc) EY-NC-ND 2016, Universitat Politècnica de València 


\section{Desarrollo de la innovación}

\subsection{Destinatarios y planificación previa:}

La experiencia se llevó a cabo en el grupo de prácticas PL-1AB1/PL-1AB2 del alumnado matriculado en la asignatura de Morfología a nivel celular y tisular, del Grado en Ingeniería Biomédica (grado mixto de la Universidad Politécnica de Valencia y la Universitat de València) en el curso académico 2015-2016. El número total de alumnos matriculados fue de 46, de los cuales, los 38 asistentes a la sesión, participaron en la gamificación realizada.

Con 6 días de antelación, se informó a los alumnos de que durante la última sesión de prácticas se destinaría parte del tiempo a la realización de diferentes actividades de repaso, reafirmación y autoevaluación de sus conocimientos basándonos en la utilización de recursos electrónicos interactivos y fundamentalmente en formato concurso. Los contenidos incluidos abarcarían 4 de los 12 temas constituyentes de la unidad de Morfología a Nivel Celular y se instó a los alumnos a preparar dichos contenidos para aprovechar la experiencia. Se prepararon 3 cuestionarios tipo test, los dos primeros con la herramienta KAHOOT y el tercero con SOCRATIVE. Aunque ambos programas se pueden utilizar para realizar actividades individuales, se diseñaron grupos para participar en los cuestionarios con KAHOOT, ya que en el formato concurso que utiliza, la ordenación de los participantes en función de sus respuestas, resulta estimulante. Además, formar pequeños equipos favorece las relaciones interpersonales de los alumnos, y al contestar desde un único dispositivo móvil, los problemas de conectividad se reducen. Por su parte, la actividad con SOCRATIVE se planificó para ser contestada de manera individual.

\subsection{Estructura de la sesión}

La sesión se estructuró en 2 actividades grupales gamificadas (KAHOOT) y 1 actividad individual de autoevaluación interactiva (SOCRATIVE). Para el desarrollo de las primeras se organizó el aula en 12 grupos de 3 alumnos y un grupo de 2 alumnos.

El primer quiz constaba de 10 preguntas de contenidos preliminares de la asignatura. Hay que tener en cuenta que una elevada proporción de nuestros alumnos no ha cursado la asignatura de Biología durante el bachillerato y aunque se recomienda que se actualicen de manera autónoma, durante las sesiones teóricas realizamos un intenso trabajo de introducción de conceptos básicos, sobre los que poder construir las sesiones. Tras la resolución de cada cuestión se procedió a repasar contextualmente los ítems sobre los que se preguntaba. El quiz comenzaba con preguntas sencillas y tiempos largos para contestar permitiendo al alumnado familiarizarse con la interfaz KAHOOT, comprobar los problemas de conectividad en caso de que los hubiera y aumentar su nivel de confianza a la hora de contestar.

El segundo quiz constaba de 15 preguntas de los contenidos de la materia y en forma y dificultad similar a las que se plantean en los exámenes oficiales de la asignatura (figura 1). De esta manera se logra un doble objetivo, positivo tanto para el alumnado como para el profesorado: por una parte pretende reducir la ansiedad de los estudiantes ante la incertidumbre de cómo se evaluará la asignatura. Por otra parte, permite al docente detectar qué tipo de enunciados resultan más complejos para su comprensión por el alumno. Tras la

(cc)) BY-Nc-ND 2016, Universitat Politècnica de València

Congreso In-Red (2016) 
resolución de cada cuestión se explicaron todas las opciones propuestas en el test y se incidió en el porqué de su veracidad o falsedad.

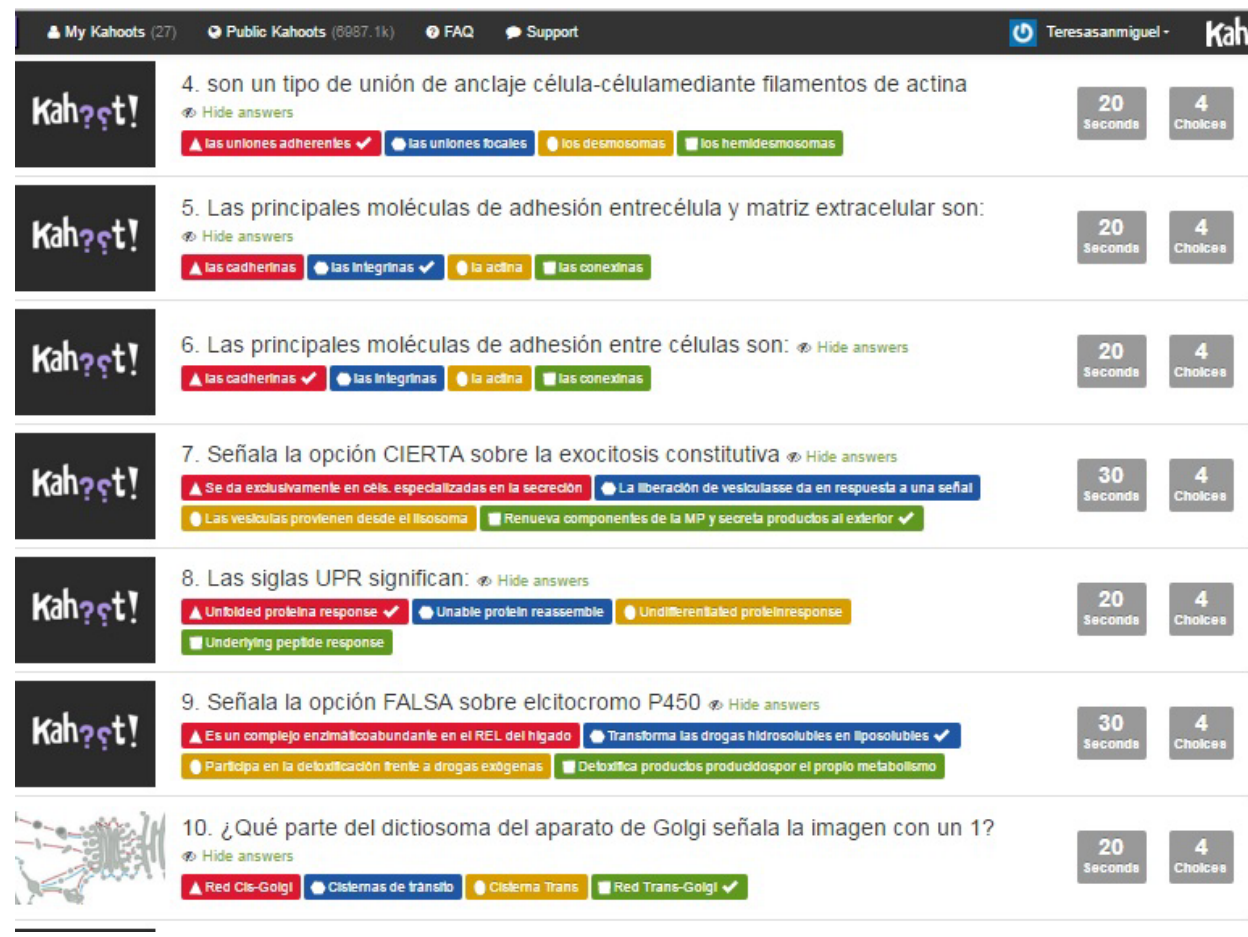

Figura 1. Representación de la interfaz que maneja el docente con el elenco de preguntas en el segundo quiz

Por último se realizó una prueba individual con el software SOCRATIVE. Constaba de 11 preguntas tipo test. SOCRATIVE permite al alumno contestar a su ritmo y le proporciona feedback inmediato, sabiendo tras lanzar su respuesta, si ésta es correcta o no. Además, permite al docente introducir junto con la corrección de la respuesta una explicación justificativa que le llega al dispositivo digital al alumno antes de seguir con la siguiente pregunta.

\subsection{Evaluación de las actividades}

Para conocer la impresión de los alumnos sobre la utilización de actividades interactivas del tipo gamificación, en la siguiente sesión teórica se les proporcionó un breve cuestionario para que valoraran la experiencia realizada, de forma voluntaria y anónima. Las preguntas incluidas fueron:

- ¿Cómo consideras este tipo de actividades innovadoras como método para afianzar conceptos? excelente-bueno-indiferente-regular-malo

- ¿Qué proporción de las preguntas desearías que se redactaran de una manera más clara y unívoca? Ninguna, menos del $10 \%$, un $10-30 \%$, un $30-60 \%$, un $60-$ $80 \%$, más del $80 \%$

(cc) EY-NC-ND 2016, Universitat Politècnica de València 
- ¿Consideras que te ha resultado útil para conocer cómo se plantean las cuestiones test de la asignatura? Muy útil-bastante útil-indiferente-poco útil-nada útil

- ¿Prefieres las actividades grupales (KAHOOT) o individuales (SOCRATIVE)?

- Valora la actividad KAHOOT. Muy útil-bastante útil-indiferente-poco útil-nada útil

- Valora la actividad SOCRATIVE. Muy útil-bastante útil-indiferente-poco útil-nada útil

- ¿Cuál es tu nivel de satisfacción con las experiencias de la práctica? Muy satisfecho-Satisfecho-Neutral-Insatisfecho-muy insatisfecho

\title{
4. Resultados
}

Desde nuestro punto de vista, la experiencia fue muy positiva, la acogida por parte de los estudiantes fue excelente ya que el $100 \%$ de los presentes, participaron voluntariamente en los concursos-KAHOOT y en la autoevaluación interactiva-SOCRATIVE.

La propuesta de realizar una actividad diferente con componente lúdico, motivó la implicación de los estudiantes: el $68,4 \%$ de los participantes refirió haber repasado los contenidos que se indicaron para desarrollar la actividad mejor.

El primer quiz que pretendía garantizar el conocimiento de cuestiones básicas sobre la biología de la célula, proporcionó una tasa de aciertos global del $84,2 \%$, con 3 de 10 preguntas con un $100 \%$ de aciertos y ninguna por debajo del $60 \%$. El segundo quiz mostró resultados inferiores, con un $56 \%$ de respuestas correctamente resueltas, si bien permitió remarcar los aspectos clave de las cuestiones planteadas.

La autoevaluación mostró un $68,1 \%$ de aciertos globales. Con independencia de los resultados obtenidos por cada grupo o individuo en las diferentes pruebas, el $87 \%$ de los alumnos encontraron que la actividad era una forma buena-excelente para afianzar los conceptos de la materia, por lo que consideramos un éxito su aplicación en este grupo (figura 2).

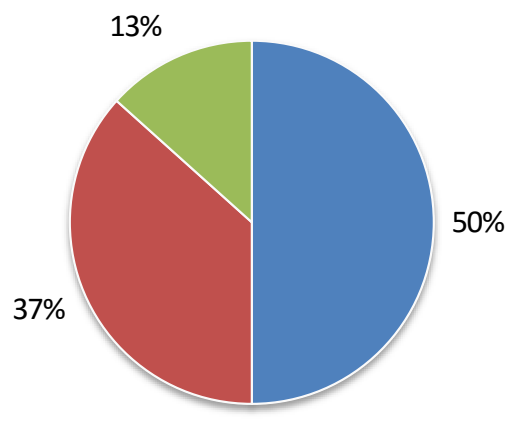

\author{
- Excelente \\ $\square$ Bueno \\ $\square$ Indiferente \\ 口 Regular \\ \alo
}

Figura 2. Valoración de la actividad innovadora como método para afianzar conceptos

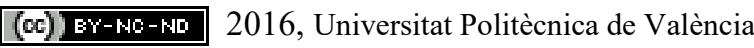

Congreso In-Red (2016) 
El desarrollo de estas actividades permitió detectar problemas para la comprensión de ciertos conceptos por parte de los alumnos; las herramientas utilizadas proporcionaron información inmediata sobre el éxito de las preguntas, permitiendo que analicemos aquellas que peor se han contestado. No obstante, mayor importancia tuvo la detección de enunciados que pueden no ser óptimos para que un estudiante, a pesar de conocer los contenidos, sea capaz de seleccionar la opción correcta en el tipo test. A la pregunta sobre la proporción de preguntas que los estudiantes piensan que podrían estar redactadas de una manera unívoca, más de la mitad de los alumnos encuestados encontraron que podrían elaborarse mejor menos del $10 \%$ de las preguntas (figura 3). Aunque los resultados son buenos merece la pena repasar aquellas en las que se han presentado mayores dificultades y formularlas de nuevo.

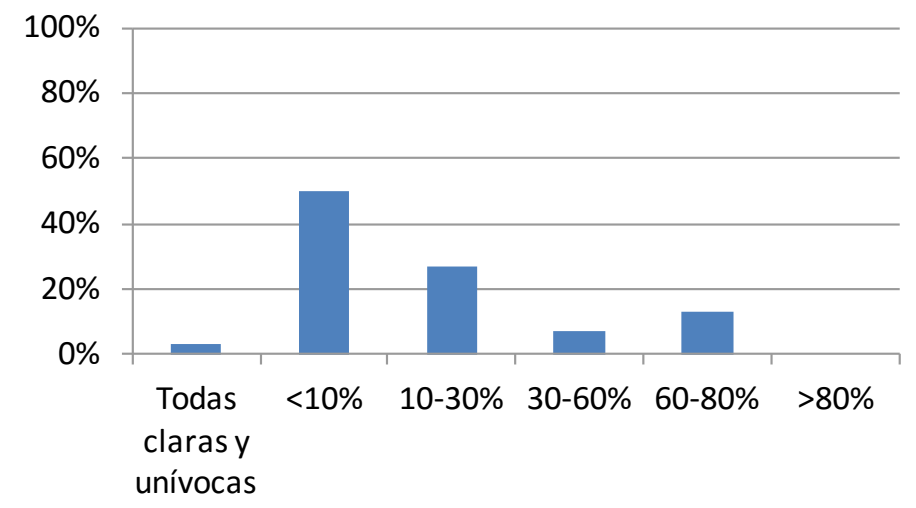

Figura 3.Impresión de los alumnos sobre la proporción de cuestiones susceptibles de una redacción más clara

Por otra parte, más allá del formato de redacción de los enunciados, el factor "sorpresa", o qué puntos considera el docente más relevantes, así como la manera en que enfoca las cuestiones, es fuente de ansiedad para los alumnos, sobre todo en su primer curso de estudios universitarios. Por esta, razón preguntamos a los participantes si encuentran útil esta actividad para conocer la forma de evaluar y reducir así su incertidumbre ante el examen y el $94 \%$ de los encuestados coincide en que le ha resultado entre bastante y muy útil (figura 4).

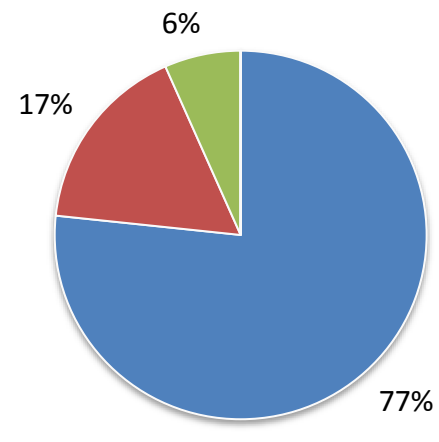

\author{
$\square$ Muy útil \\ - Bastante útil \\ $\square$ Indiferente \\ $\square$ Poco útil \\ $\square$ Nada útil
}

Figura 4. Utilidad de las actividades propuestas para conocer cómo se plantea la evaluación de la asignatura

(cc) EY-NC-ND 2016, Universitat Politècnica de València

Congreso IN-RED (2016) 
Para conocer la impresión de los alumnos sobre la utilización de actividades interactivas de tipo gamificación en el aula, además de su satisfacción global que ya hemos comentado, les pedimos que valorasen del 1 al 5 de manera conjunta la diversión y utilidad de cada una de las herramientas utilizadas. Entre actividades grupales o individuales, el 70\% prefirió las actividades grupales (figura 5). Dentro de las valoraciones específicas de cada uno de los programas utilizados, que fueron positivamente valorados por los estudiantes, la prueba individual desprendió un nivel de indiferencia mayor (27\%).

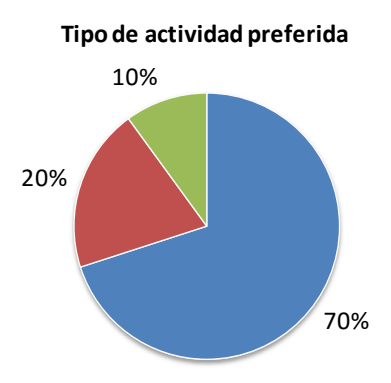

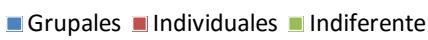

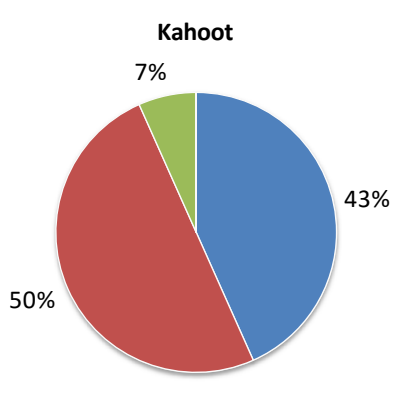

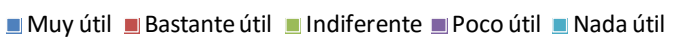

Figura 5. Preferencia sobre las actividades propuestas y utilidad que perciben los alumnos sobre ellas.

Por último, el nivel de satisfacción de los alumnos fue muy alto según los resultados del cuestionario de evaluación de la actividad, con un $90 \%$ de los encuestados satisfechos o muy satisfechos, concretamente el $37 \%$ se mostró muy satisfecho con la experiencia (figura 6). Todas las actividades se desarrollaron en 35 minutos dentro de la última sesión práctica de la Unidad; este tiempo fue suficiente para incluir también aclaraciones a cada una de las cuestiones planteadas en los dos quizes con KAHOOT y para contextualizarlas en su ámbito morfológico-funcional, lo que enfatiza la relativa sencillez con que podría incluirse alguna de las actividades de forma sistemática en la programación de aula.

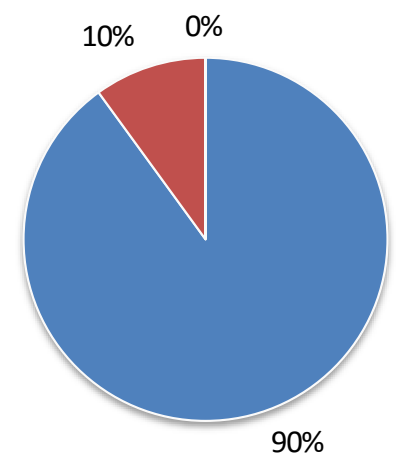

\author{
口Satisfecho/Muy satisfecho \\ $\square$ Neutral \\ $\square$ Insatisfecho
}

Figura 6. Satisfacción que refieren los alumnos que participaron en la actividad

(cc) BY-NC-ND 2016, Universitat Politècnica de València

Congreso In-Red (2016) 


\section{Conclusiones}

En esta experiencia, el primer elemento a destacar fue la implicación de los estudiantes que, con mayor o menor rendimiento, refirieron haber dedicado tiempo a preparar voluntatariamente la sesión, conscientes de los posibles beneficios que les reportaría. Esto enfatiza por una parte la idea del "divide y vencerás", pues el planteamiento incluía solo una pequeña porción de lo explicado en el aula, animando a los alumnos a mejorar su dominio sobre parte de la asignatura y reduciendo así la sensación de carga total. Por otra parte, revela lo sencillo que puede llegar a ser romper con la estructura clásica de las sesiones: pruebas de apenas 10 cuestiones permiten afianzar una amplia variedad de procesos y conceptos.

Desde nuestro punto de vista, fue excepcional la estimulación que mostraron los estudiantes: tras la primera pregunta de KAHOOT con un $100 \%$ de aciertos, se aplaudían y animaban entre ellos, creando un clima de confort que no decaía cuando comenzaron a aparecer las primeras dificultades.

Prácticamente el 90\% encontró que estas actividades resultan positivas para afianzar conceptos. Además, una elevadísima proporción de los participantes (el 94\%) se siente más seguro con la asignatura tras descubrir, mediante el juego, el tipo de razonamientos que implicará la forma de evaluar en el examen, favoreciendo un comportamiento que ha de cambiar de memorístico a comprensivo, y demostrándoles la necesidad de "conocer" para "entender". Aunque todavía no disponemos de resultados comparables, confiamos en poder confirmar una mejoría global en las calificaciones cuando finalice el curso académico.

Más de dos tercios de los participantes prefirió las actividades grupales frente a las individuales. Cuando valoramos el el grado de satisfacción con ambos tipos de actividades, fue sutilmente más elevado en las grupales, pero lo más destacable fue la tasa de indiferencia que supuso la actividad individual: utilizada como actividad de autoevaluación en el aula, enfatiza que lo más motivante para el alumnado durante la sesión, era poder debatir con sus compañeros de grupo las posibles respuestas y así interiorizar los contenidos, alcanzando un mejor nivel de comprensión y, no menos importante, haciéndolo de una manera divertida. Además, estos resultados también inciden en el papel como director-motivador del juego que desempeña el docente.

Por todo lo expuesto podemos concluir que la experiencia ha resultado muy positiva, tanto para nuestro alumnado como para nosotros como docentes. Nos ha proporcionado valiosa información sobre como continuar implementando las nuevas tecnologías aplicadas a la docencia universitaria, mejorar la implicación de nuestros alumnos, reducir sus temores ante los sistemas de evaluación y aumentar su motivación hacia al aprendizaje.

\section{Bibliografía}

CONTRERAS, L.C. Y RODRÍGUEZ, J.M. (2004). "La formación del profesorado universitario en el marco del Espacio Europeo de Educación Superior”. Revista Comunicación y Pedagogía, n95, p.611 .

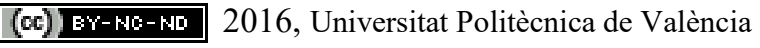


CORTIZO PÉREZ, JC. CARRERO GARCÍA, F. MONSALVE PIQUERAS, B. VELASCO COLLADO, A. DÍAZ DEL DEDO, LI. y PÉREZ MARTÍN, J. (2011). "Gamificación y Docencia: Lo que la Universidad tiene que aprender de los Videojuegos". En: Retos y oportunidades del desarrollo de los nuevos títulos en educación superior. VIII Jornadas Internacionales de Innovación Universitaria. Universidad Europea de Madrid (2011 Madrid). Disponible en $<$ http://hdl.handle.net/11268/1750>

KAHOOT. Wang, A.I. (2013). Norwegian University of Science and Technology (NTNU). Trondheim, Noruega (Høgskoleringen 1, 7491 Trondheim, Noruega). <https://kahoot.it/> [Consulta: 1 de abril de 2016]

KAPP, K.M. (2012). The Gamification of Learning and Instruction: Game-Based Methods and Strategies for Training and Education. San Francisco, CA: John Wiley

MARTÍNEZ GONZÁLEZ J.A. (2011). "La motivación para aprender en el espacio europeo de educación superior". Cuadernos de Educación y Desarrollo, Vol. 3 (25). http://www.eumed.net/rev/ced/25/jamg.htm [Consulta: 01-04-2016].

MARTOS MOLINA, M. y PULIDO FERNÁNDEZ, J I. (2011) "Retos para la gestión turística de los destinos urbanos culturales en España”. López, D. (coord.) En: Renovación de destinos turísticos consolidados. Congreso de Turismo Universidad y Empresa (13. 2010. Castellón de La Plana). Valencia: Tirant lo Blanch. 223-256

MAURICIO, M.D, SERNA, E y VALLES, S.L. (2015). "Experiencias en la aplicación de la gamificación en $1^{\circ}$ Curso de Grado de Ciencias de la Salud". En: Congreso de Innovación Educativa $y$ Docencia en Red. Universitat Politècnica de València (2015 Valencia). Disponible en $<$ http://ocs.editorial.upv.es/index.php/INRED/>

NMC and the EDUCAUSE Learning Initiative (ELI). NMC Horizon Report - 2016 Higher Education Edition. Disponible en: $\quad<$ http://www.nmc.org/publication/nmc-horizon-report-2016-highereducation-edition $>$ [Consulta: 1 de abril de 2016]

PINTOR HOLGUÍN, E., GARGANTILLA MADERA, P., HERREROS RUIZ VALDEPEÑAS, B. Y LÓPEZ DEL HIERRO, M (2014). "Kahoot en docencia: una alternativa práctica a los clickers". En: XI Jornadas Internacionales de Innovación Universitaria. Educar para transformar. Universidad Europea de Madrid (2014 Madrid). Disponible en <http://hdl.handle.net/11268/3603>

PIÑEIRO-OTERO, T. Y COSTA-SÁNCHEZ, C. (2015). "ARG (juegos de realidad alternativa). Contribuciones, limitaciones y potencialidades para la docencia universitaria". Comunicar, Vol. 44(22), pp.141-148

SOCRATIVE. MasteryConnect. Salt Lake City, UT (222 South Main, Suite 200. Salt Lake City, UT 84101, USA). <www.socrative.com/> [Consulta: 1 de abril de 2016]

TEJEDOR, F.J. Y GARCÍA-VALCÁRCEL, A. (2007). "Causas del bajo rendimiento del estudiante universitario (en opinión de los profesores y alumnos). Propuestas de mejora en el marco del EEES". Revista de Educación, vol. 342, p. 443-473

ZICHERMANN, G. Y CUNNINGHAM, C. (2011). Gamification by Design: Implementing Game Mechanics in Web and Mobile Apps. Cambridge, MA: O'Reilly Media

(cc) EY-NC-ND 2016, Universitat Politècnica de València

Congreso In-Red (2016) 